\title{
Prenatal Diagnosis of Heart Rabdomioma. A Case Report
}

\author{
Illia $\mathrm{R}^{1}$, Engel $\mathrm{H}^{2}$, Guallan $\mathrm{F}^{1}$, Faganello $\mathrm{A}^{3}$ and Lopez $\mathrm{SV}^{4}$ \\ ${ }^{1}$ Chief Residents of Gynecology Service, Hospital Alemán, Argentina \\ ${ }^{2}$ Staff physician at Gynecology Service, Hospital Alemán, Argentina \\ ${ }^{3}$ Staff physician at Imagenology Service, Hospital Alemán, Argentina \\ ${ }^{4}$ Staff physician Genetic Service, CEMIC, Argentina
}

*Corresponding author: Illia R, Department of Obstetrics, Buenos Aires University, Fellow ACOG Buenos Aires Argentine Section, Chief of Obstetrics Service, Hospital Alemán, Buenos Aires, Argentina.

Received Date: August 31, 2018

Published Date: September 21, 2018

\begin{abstract}
We describe a case of a heart tumor diagnosed by ultrasound in a 20 week's gestational age fetus with presumptive diagnosis of heart rabdomioma. The fetus was delivered by cesarean section because a previous section at 38 weeks of gestational age. At the evaluation of the new born, the diagnosis of rabdomioma was confirmed by echocardiogram. We report of a case of prenatal rabdomioma associated to maternal diagnosis of tuberous sclerosis.
\end{abstract}

Keywords: Tuberous sclerosis; Prenatal rabdomioma; Fetal heart tumor

\section{Introduction}

The Tuberous Sclerosis (TS) (OMIM \#191100; \# 613254), is a genetic disease, autosomic dominant, multisistemic, characterized by the presence of hamartoms in brain, skin, heart, kidney, lungs and neuropsyquiatric symptoms. The prevalence of the disease is $1: 10000$. Caused by the mutation in one of the gene TSC1, which is localized in the long arm of the chromosome 9 or TSC2 (localized in the short arm of the chromosome 16). The disease has variable expression and it can start at any time of the life [1].

\section{Case Report}

Patient 35 years old, second pregnancy with a previous cesarean section. We do not have familiar background because she was adopted. She has a daughter 5 years old apparently healthy by the time of the present pregnancy. As a personal background, the patient has remotion of nose angiofibroms in 2008, cauterization of skin macular lesions and embolization of kidney angiolipomes of 3 and $4 \mathrm{~cm}$ in 2015.

The pregnancy was uneventful, with normal screening 11-14 weeks. She came to prenatal control monthly and a fetal scan was performed at 20 weeks of gestational age. The anatomical measures were normal for gestational age with a male fetus growing in a 75 percentile, normal amniotic fluid. Heart with four chambers and 3 vessels with a homogeneous hiperechogenic focus in left ventricle, apical zone, size $5 \times 4 \mathrm{~mm}$ with growth toward ventricle cavity, could be a rabdomioma.

At 25 weeks of gestational age, a new ultrasound control showed a persistent hiperechogenic image in apical zone of left ventricle with a size of $11 \times 9 \mathrm{~mm}$. A new control at 32 weeks showed the same image but this time the size is $20 \times 11 \mathrm{~mm}$. At 36 weeks of gestational age, an ultrasound evaluation showed a fetus growing in 25 percentile, amniotic fluid normal, hiperechogenic mass in left ventricle of $22 \times 1 \mathrm{~mm}$. The Doppler evaluation was normal with arterial and venous flows preserved.

A fetal echocardiogram was done at 36 weeks of gestational age. It shows a homogeneous tumor in left ventricle which is compatible with rabdomioma. Laminar flows in input and output tracts. Fetal heart rate of 130 by minute, regular. Ventricle function conserved. No dilated cavities. 
A cesarean section was scheduled for 38 weeks because a previous scar. It was delivered a male new born, 2600g, Apgar 8/9 (Figure 1 \& Figure 2).

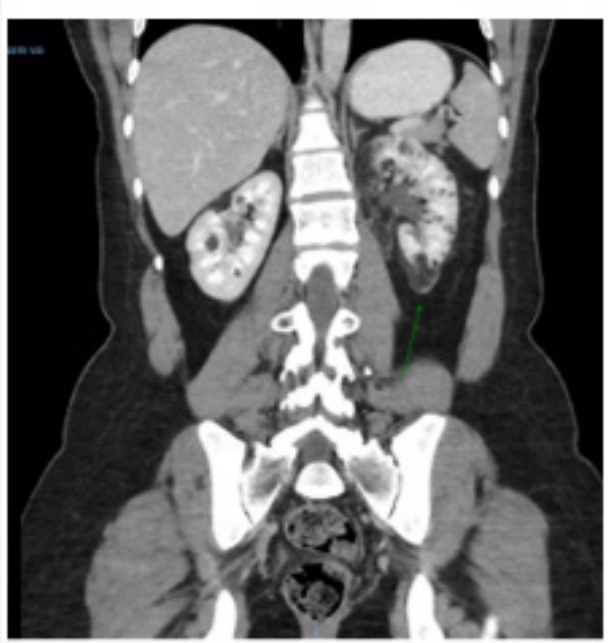

Figure 1: Abdominal TC showing angiolipomes in left kidney.

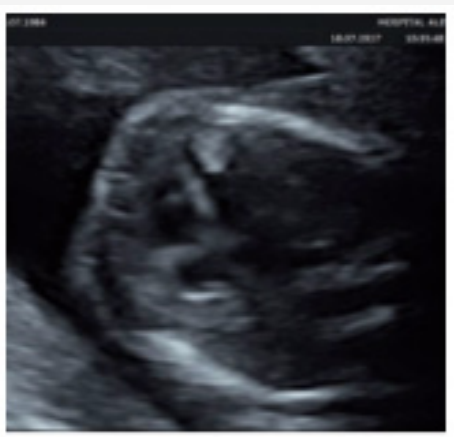

Figure 2: Fetal scan with a heart with four chambers showing a cardiac tumor in apical zone of left ventricle.

A diagnosis of rabdomioma was confirmed by neonatal ultrasound which informed: big tumoral mass in left ventricle of 27 $\mathrm{mm}$, ventricle function conserved. Abdominal and brain ultrasound were normal. A cardiac Holter at the second day of life informed sinusal rim preserved without neither AV blocks nor sinusal automatism depression.

\section{Comments}

Among the most common clinical presentations of TS are the pigmentary and proliferative lesions of the skin, brain malformations, heart rabdomiomas, kidney angiolipomes and lung linfangioleiomiomatosis. The great morbimortality is determinate by the brain function affection and a high prevalence of refract epilepsy. The kidney lesions, generally angiomiolipomes, may cause clinical problems because hemorrhage or by compression and replacement of healthy tissue that finally could produce kidney failure. These patients also can develop kidney cysts and kidney carcinoma. The skin lesions include melanotic macula, facial angiofibromes and nevus of connective tissue. About 10 to $30 \%$ of cases of TS are due to mutations of gene TSC1, the frequency of cases due to mutations of gen TSC2 is $69 \%$. The mutations of TSC2 are associated to more severe disease.

The diagnosis is based in clinical diagnostic criteria agreed at international level (last revision in 2012) [2] that include the most frequent manifestations of the disease. The diagnosis is confirmed by molecular technique identify clearly a pathogenic mutation in TSC1 or TSC2, detectable in almost $80 \%$ of cases. In the other $20 \%$ of cases is due probably to a mosaicism.

In reference of the treatment of the disease, the different manifestations need a multidisciplinary management, from the treatment of the epilepsy to the treatment of a kidney or lung failure. It was recentlly approved the use of everolimus, a drug with inhibitory effect of the mTOR, to decrease the growth of brain tumors and kidney angiolipomes [3]. It is expected that in the near future their use could be extended to treat other manifestations of the disease.

\section{Conclusion}

The early diagnosis of heart rabdomioma during the prenatal period is the upmost importance for the follow up and prognosis of the pregnancy and for the inquiry of TS, because the patients with heart rabdomioma have a risk of $75-80 \%$ to suffer TS [4]. In the case of a postnatal clinical or molecular diagnosis is done, a strict follow up of the new born is wise with dermatological, odonatological and ophthalmological control. Besides there are to control the blood pressure, perform an RMN of brain, EEG, ECG, tests of lung function, TC of thorax and abdomen and check the kidney function. Performing an early diagnosis of TS in pediatric patients before the start of seizures, allow them to develop a less severe epilepsy and have a better development.

\section{References}

1. Curatolo P, Bombardieri R, Jozwiak S (2008) Tuberous sclerosis. Lancet 372(9639): 657-668.

2. Northrup H, Krueger DA (2013) Tuberous sclerosis complex diagnostic criteria update: recommendations of the 2012 International tuberous sclerosis complex consensus conference. Pediatr Neurol 49(4): 243-254.

3. Mlczoch E, Hanslik A, Luckner D, Kitzmüller E, Prayer D, et al. (2015) Prenatal diagnosis of giant cardiac rhabdomyoma in tuberous sclerosis complex: a new therapeutic option with everolimus. Ultrasound Obstet Gynecol 45(5): 618-621.

4. Chung CWT, Lawson JA, Sarkozy V, Riney K, Wargon O, et al. (2017) Early detection of tuberous sclerosis complex: An opportunity for improved neurodevelopmental outcome. Pediatr Neurol 76: 20-26. 